1 Universidade de São Paulo (USP), Faculdade de Saúde Pública (FSP) - São Paulo (SP), Brasil.

kincha@yahoo.com.br

2 Universidade de São Paulo (USP), Faculdade de Saúde Pública (FSP) - São Paulo (SP), Brasil. amandafarias_23@hotmail. com

3 Universidade de São Paulo (USP), Faculdade de Saúde Pública (FSP) - São Paulo (SP), Brasil. cervato@usp.br

\section{Planejamento de grupos operativos no cuidado de usuários de serviços de saúde: integrando experiências}

\author{
Planning of operative groups in the care of health services users: \\ integrating experiences
}

Kellem Regina Rosendo Vincha' ${ }^{\mathbf{1}}$ Amanda de Farias Santos², Ana Maria Cervato-Mancuso ${ }^{\mathbf{3}}$
RESUMO O objetivo deste estudo foi apresentar um panorama do planejamento de grupos operativos realizados com usuários de serviços de saúde. Realizou-se uma revisão na base de dados Scientific Electronic Library Online (SciELO). Nos 12 estudos analisados, a teoria de grupo operativo teve como base cinco autores diferentes. Os grupos pretenderam prevenir doenças/agravos e tratar doenças, porém o processo destacou a promoção da saúde associada à educação em saúde e à educação alimentar e nutricional. A observação foi o método de avaliação mais aplicado, entretanto, poucos estudos usaram indicadores de avaliação. O panorama construído poderá auxiliar profissionais no momento de planejar e desenvolver seus grupos.

PALAVRAS-CHAVE Revisão. Planejamento em saúde. Promoção da saúde. Processos grupais.

ABSTRACT The objective of this study was to present a panorama of the planning of operative groups carried out with health services users. A review was conducted in the Scientific Electronic Library Online (SciELO) database. In the 12 studies analyzed, the operative group theory was based on five different authors. The group aimed to prevent diseases/grievances and treat diseases, but the process highlighted the promotion of health associated with health education and food and nutrition education. The most common method of used evaluation was observation, however, few studies used evaluation indicators. The built panorama can help professionals in the planning and development of their own groups.

KEYWORDS Review. Health planning. Health promotion. Group processes. 


\section{Introdução}

O grupo, como modalidade de cuidado coletivo à população, tem se tornado frequente nos serviços de saúde, devido ao seu reconhecimento enquanto prática de educação em saúde. $\mathrm{O}$ cuidado em grupo envolve, a partir de relações interpessoais, a constituição de subjetividade e do psiquismo, a elaboração do conhecimento e a aprendizagem em saúde (AFONSO; COUTINHO, 2010; FERREIRA NETO; KIND, 2010).

Uma teoria de grupo legitimada na área da saúde é o Grupo Operativo (GO), que foi elaborada por Pichon-Rivière, psiquiatra e psicanalista, na década de 1940 (MENEZES; AVELINO, 2016). O objetivo do GO é promover um processo de aprendizagem para os participantes, o qual é compreendido como uma leitura crítica da realidade, uma atitude investigadora, uma abertura para as dúvidas e para as novas inquietações. Este processo coloca em evidência a possibilidade de uma nova elaboração de conhecimento, de integração e de questionamentos acerca de si e dos outros (AFONSO; COUTINHO, 2010).

Desse modo, o GO na área da saúde visa não apenas conceber as condições de saúde/ doença por meio da transmissão de conhecimento - abordagem utilizada por muitos profissionais da saúde (MELO, 2013; VINCHA ET AL., 2014) -, mas também transformar o conhecimento em atitude, a partir das necessidades e da realidade dos participantes. Esta transformação ocorre por meio do vínculo, da comunicação e do protagonismo de todos. Por isso, significados, sentimentos, relações, expectativas e experiências individuais são implicados na ação (AFONSO; COUTINHO, 2010; PICHON-RIVIĖRE, 2009).

O GO, como processo de aprendizagem, pode ser utilizado na prevenção de doenças e no tratamento de cuidados específicos, porém ele está cada vez mais presente na promoção da saúde (DUTRA; CORRÊA, 2015; MENEZES; AVELINO, 2016). Esta mudança de perspectiva decorre de as ações promotoras da saúde possuírem como objetivo o estímulo do empoderamento, a capacidade para a tomada de decisão e a autonomia, de indivíduos e coletividades, por meio do desenvolvimento de habilidades pessoais e de competências em promoção e defesa da saúde e da vida (BRASIL, 2014C), o que vai ao encontro da teoria do GO. Neste sentido, de acordo com a Política Nacional de Promoção da Saúde (BRASIL, 2014C), essas ações buscam proporcionar a equidade e a melhoria das condições e dos modos de viver, reduzindo vulnerabilidades e riscos à saúde decorrentes de determinantes sociais, econômicos, políticos, culturais e ambientais.

O desenvolvimento do GO depende de um clima particular, logo, os profissionais planejam a intervenção com o intuito de transformar uma situação em um campo propício de aprendizagem (PICHON-RIVIĖRE, 2009). Ao compreender o GO como prática de educação em saúde, o planejamento, realizado de forma sistematizada ou não, pode ser considerado uma ferramenta de intervenção dos profissionais da saúde (CERVATO-MANCUSO, 2011). Neste sentido, o planejamento do GO, assim como ações educativas e oficinas de grupo na área da saúde, é composto pelos seguintes elementos: definição do referencial teórico; análise das demandas de saúde da população atendida; elaboração do objetivo do grupo; identificação da tarefa; análise de temas pertinentes; escolha de estratégias educativas; e avaliação (AFONSO, 2010; CERVATO-MANCUSO, 2011).

O referencial teórico é a fundamentação teórica do grupo, na qual os profissionais da saúde se apoiam para conduzir a intervenção. Ela pode ser fundamentada em um ou mais autores de GO. A análise das demandas de saúde visa identificar os problemas de saúde da população e seus fatores causais, os quais fornecem suporte para a elaboração do objetivo. Com o objetivo elaborado, dado que ele é fixo, tem-se um guia para o desenvolvimento do grupo (CERVATO-MANCUSO, 2011), bem como se podem estabelecer regras de organização, como: local de encontro; dias e horário; frequência dos encontros; número de participantes; e profissionais coordenadores da ação (AFONSO, 2010). 
Para a concretização do objetivo, o GO centra-se na tarefa, que é o fazer conjunto dos participantes, a partir de um esquema referencial comum. É por meio da tarefa, e pela tarefa, que os participantes estabelecem redes de comunicação e processos de aprendizagem, que ocorrem por objetivos conscientes e por relações internas, inconscientes, no grupo e do grupo (CASTANHO, 2012; PICHON-RIVIĖRE, 2009). Na área da saúde, o GO que objetiva a aprendizagem tem como dispositivos para a realização da tarefa os temas de discussão, pois apoiado neles, suscitam-se e mobilizam-se sentimentos, pensamentos e ações nos participantes (CASTANHO, 2012). Os temas podem ser identificados antes do início do grupo, na análise das demandas de saúde, o que oportuniza a existência de um cronograma definido, ou podem ser identificados a partir das necessidades emergidas no decorrer do grupo. Eles podem ser trabalhados com o uso de estratégias educativas, ou seja, palestras, vídeos, jogos, fotos, músicas e dinâmicas, entre outras (AFONSO, 2010).

A avaliação do grupo, último elemento do planejamento, deve conter uma análise dos participantes e uma análise do processo grupal. Desta forma, associam-se indicadores individuais, comumente conhecidos na área da saúde, e coletivos, sendo que, em ambos, as abordagens quantitativas e qualitativas podem ser exploradas (FERREIRA NETO; KIND, 2011; GAYOTTO, 2003). O processo grupal pode ser avaliado por seis vetores de avaliação, formulados por Pichon-Rivière (2009), que são: pertença; pertinência; comunicação; cooperação; aprendizagem; e tele.

Dado o reconhecimento do GO na área da saúde como uma teoria que favorece o cuidado integral dos participantes e que viabiliza a efetivação de grupos como espaços educativos em saúde, o presente estudo teve por objetivo apresentar um panorama do planejamento de GO realizados com usuários de serviços de saúde.

\section{Métodos}

Este estudo constitui uma medição de produções científicas na área da saúde sobre GO. Esta medição denomina-se bibliometria, estudo que quantifica a comunicação escrita, que visa ao tratamento quantitativo e à organização, à classificação e à avaliação das características das publicações (BUFREM; PRATES, 2005).

Realizou-se uma busca na base de dados Scientific Electronic Library Online (SciELO), com o uso das palavras-chave 'grupo' e 'operativo'. Inicialmente, foram incluídos todos os artigos que se encontravam disponíveis durante o período da busca: setembro a dezembro de 2015. Para a seleção, foram utilizados os seguintes critérios de inclusão: artigos publicados nos idiomas português, espanhol e inglês; e artigos publicados entre os anos 2005 e 2015.

Nessa seleção, 51 artigos foram elegidos, sendo que, a partir da leitura desses na íntegra, realizaram-se as exclusões, de acordo com os critérios apresentados na figura 1: estudo que não utilizou o GO como intervenção; estudo não realizado em serviços de saúde; e estudo que não envolveu exclusivamente usuários como participantes do grupo. Assim, foram excluídos 29 pelo uso da palavra 'operativo', com significados diferentes da teoria; sete pela utilização da teoria na área do ensino; e três pelo envolvimento de profissionais da saúde como participantes do grupo. Portanto, os estudos que utilizaram o GO no cuidado de usuários da saúde totalizaram-se em 12. 
Figura1. Fluxograma de inclusão e exclusão de estudos sobre grupo operativo aplicado com usuários de serviços de saúde. São Paulo (SP), 2016

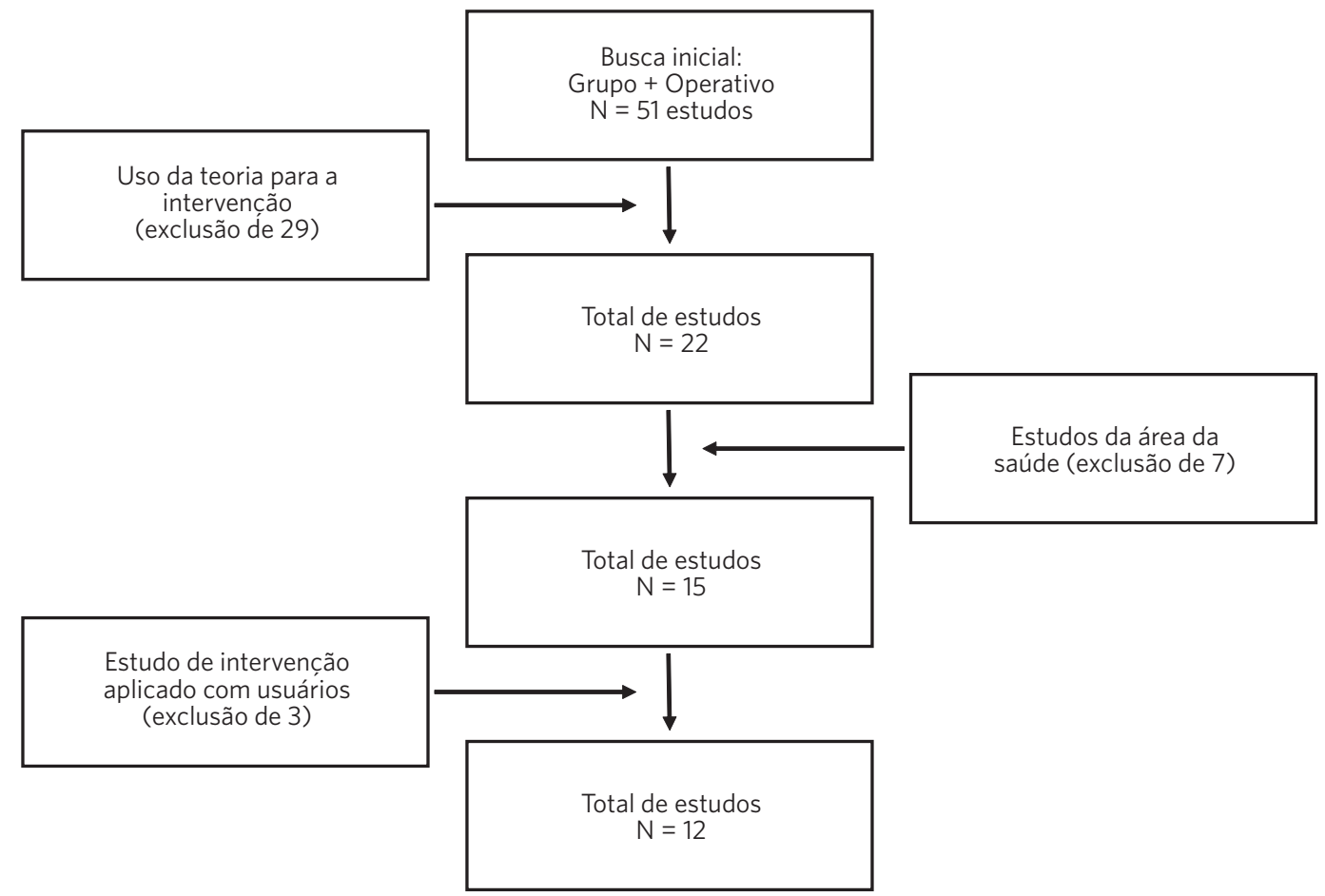

Fonte: Elaboração própria.

A bibliometria no presente estudo foi aplicada com o propósito de construir um panorama do planejamento dos GO. Para isso, buscou-se identificar os elementos do planejamento descritos nos estudos, quer dizer, os referenciais teóricos de GO, os objetivos dos grupos, os temas abordados, as estratégias educativas utilizadas e as avaliações. Quanto aos referenciais teóricos, houve a sistematização e a hierarquização dos autores, conforme o número de vezes em que foram referenciados, sendo que foram considerados aqueles citados ao menos em dois estudos. O objetivo do grupo foi classificado, de acordo com a intencionalidade, em: promoção da saúde; prevenção de riscos e doenças; e tratamento de cuidados específicos. E para a avaliação buscou-se identificar o método e o indicador empregados.

\section{Resultados}

Os estudos analisados apresentaram as seguintes características, como se vê no quadro 1: publicados entre os anos 2007 e 2015; 10 na língua portuguesa e dois em espanhol; 11 conduzidos no Brasil e um no México. No Brasil, destacou-se o estado do Rio Grande do Sul, seguido por Minas Gerais. Em relação ao tipo de estudo, identificaram-se 11 qualitativos, sendo cinco do tipo estudo de caso/relato de experiência; quatro descritivos, exploratórios ou analíticos; dois etnográficos; e um estudo quali-quantitativo de intervenção controlada. As populações investigadas foram, sobretudo, usuários de álcool e outras drogas, e portadores de doenças crônicas, como diabetes mellitus, hipertensão arterial e obesidade. Nos contextos das investigações, incluíram-se hospitais, Unidades Básicas de Saúde (UBS) e Centros de Apoio Psicossocial. 


\begin{tabular}{|c|c|c|c|c|}
\hline Autores e ano & Local & Tipo de estudo & População & Contexto do estudo \\
\hline Jardim et al. (2007) & Rio Grande do Sul/Brasil & Qualitativo, estudo de caso & Pais e filhos & Hospital \\
\hline Soares e Ferraz (2007) & Minas Gerais/Brasil & Qualitativo, estudo de caso & $\begin{array}{l}\text { Portadores de diabetes } \\
\text { mellitus }\end{array}$ & Hospital \\
\hline Pereira et al. (2007) & Rio Grande do Sul/Brasil & $\begin{array}{l}\text { Qualitativo, relato de expe- } \\
\text { riência }\end{array}$ & Mulheres & Unidade Básica de Saúde \\
\hline Colosio et al. (2007) & São Paulo/Brasil & $\begin{array}{l}\text { Quali-quantitativo, inter- } \\
\text { venção controlada }\end{array}$ & $\begin{array}{l}\text { Homens que fazem sexo } \\
\text { com homens }\end{array}$ & $\begin{array}{l}\text { Centro de Testagem e } \\
\text { Aconselhamento }\end{array}$ \\
\hline Favoreto e Cabral (2009) & Rio de Janeiro/Brasil & $\begin{array}{l}\text { Qualitativo com análise da } \\
\text { narrativa }\end{array}$ & $\begin{array}{l}\text { Portadores de doenças } \\
\text { crônicas }\end{array}$ & Hospital \\
\hline Almeida e Soares (2010) & Minas Gerais/Brasil & Qualitativo, etnográfico & $\begin{array}{l}\text { Portadores de diabetes } \\
\text { mellitus }\end{array}$ & Unidade Básica de Saúde \\
\hline $\begin{array}{l}\text { Figueroa, Guerra e Gallegos } \\
\text { (2010) }\end{array}$ & Jalisco/México & Qualitativo, etnográfico & Mulheres & Comunidade \\
\hline Cassol et al. (2012) & Rio Grande do Sul/Brasil & Qualitativo, descritivo & $\begin{array}{l}\text { Usuários de álcool e outras } \\
\text { drogas }\end{array}$ & Hospital \\
\hline Jorge et al. (2012) & Goiás/Brasil & Qualitativo & $\begin{array}{l}\text { Usuários de álcool e outras } \\
\text { drogas }\end{array}$ & $\begin{array}{l}\text { Centro de Apoio Psicos- } \\
\text { social }\end{array}$ \\
\hline Rotoli et al. (2012) & Rio Grande do Sul/Brasil & $\begin{array}{l}\text { Qualitativo, relato de expe- } \\
\text { riência }\end{array}$ & $\begin{array}{l}\text { Usuários de álcool e outras } \\
\text { drogas }\end{array}$ & $\begin{array}{l}\text { Centro de Apoio Psicos- } \\
\text { social }\end{array}$ \\
\hline Lucchese et al. (2013) & Ceará/Brasil & $\begin{array}{l}\text { Qualitativo, descritivo e } \\
\text { exploratório }\end{array}$ & Usuários de tabaco & Unidade Básica de Saúde \\
\hline Dutra e Correa (2015) & Minas Gerais/Brasil & $\begin{array}{l}\text { Qualitativo, relato de expe- } \\
\text { riência }\end{array}$ & Cuidadores de idosos & Unidade Básica de Saúde \\
\hline
\end{tabular}

Fonte: Elaboração própria.

A partir dos elementos do planejamento dos GO constatados nos estudos, referenciais teóricos, objetivos, temas, estratégias educativas e avaliações, foi construído um panorama, apresentado na figura 2, que mostra as possibilidades existentes para este conjunto de ações, que oportunizam o cuidado em grupos de usuários por profissionais da saúde. 
Figura 2. Panorama do planejamento de grupo operativo para o cuidado de usuários de serviços de saúde, São Paulo (SP), 2016

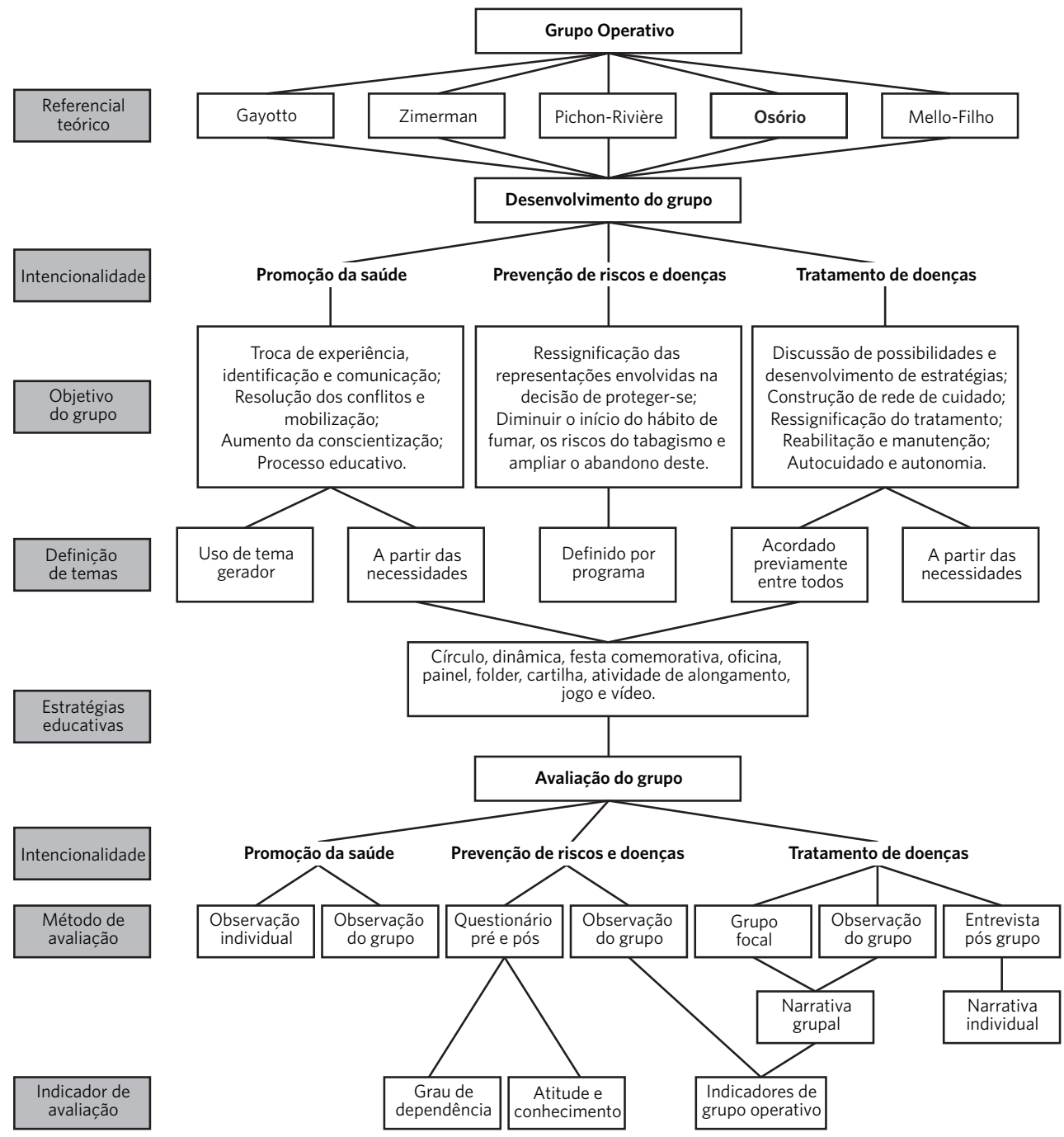

Fonte: Elaboração própria.

Em relação ao referencial teórico de GO, verificou-se a menção de cinco autores diferentes. O autor Pichon-Rivière (2009) foi referenciado nove vezes em sete estudos (ALMEIDA; SOARES, 2010; COLOSIO ET AL., 2007; DUTRA; CORRÊA， 2015; FIGUEROA; GUERRA; GALLEGOS， 2010; LUCCHESE ET AL., 2013; ROTOLI ET AL., 2012; SOARES; FERRAZ, 2007); Osório (1986), três vezes em dois estudos (ALMEIDA; SOARES, 2010; SOARES; FERRAZ, 2007); Zimerman (2000) (CASSOL ET AL., 2012; JARDIM ET AL., 2007; PEREIRA ET AL., 2007) e Gayotto (2003) (ALMEIDA SOARES, 2010; LUCCHESE ET AL., 2013; SOARES; FERRAZ, 2007) apareceram em três estudos; e Mello Filho (2011), em dois (CASSOL ET AL., 2012; FAVORETO; CABRAL, 2012). Observou-se que os estudos que usaram Zimerman denominaram os grupos como 'grupo operativo de ensino-aprendizagem' e 'grupo operativo de reflexão'. Osório e Mello Filho foram utilizados nos estudos que desenvolveram grupos para o tratamento de doenças, especialmente o diabetes mellitus. Já Gayotto foi referência para a conceitualização e a avaliação dos GO.

De acordo com os objetivos propostos 
para os grupos, dos 12 estudos analisados, foi observado que quatro tinham por intenção a promoção da saúde (DUTRA; CORRÊA, 2015; FIGUEROA; GUERRA; GALLEGOS, 2010; JARDIM ET AL., 2007; PEREIRA ET AL., 2007), sendo três deles do tipo estudo de caso/relato de experiência; dois, a prevenção de riscos e doenças (COLOSIO ET AL., 2007; LUCCHESE ET AL., 2013); e seis, o tratamento de cuidados específicos (ALMEIDA; SOARES, 2010; CASSOL ET AL., 2012; FAVORETO; CABRAL, 2009; JORGE ET AL., 2012; ROTOLI ET AL., 2012; SOARES; FERRAZ, 2007). Destaca-se, nos objetivos dos grupos de prevenção e de tratamento, a presença da ressignificação, da possibilidade, do encontro de soluções, da construção de rede de cuidado e do fortalecimento do autocuidado e da autonomia.

Sobre as regras de organização para o alcance dos objetivos dos grupos, dos estudos que as apresentaram, o número de participantes por encontro variou de 5 a 25 ; o número de encontros por grupo, de 5 a 24; e a frequência dos encontros, entre semanal e mensal. Os profissionais coordenadores foram, na sua maioria, enfermeiros (LUCCHESE ET AL., 2013; ROTOLI ET AL., 2012; SOARES; FERRAZ), seguidos por psicólogos (COLOSIO ET AL., 2007; DUTRA; CORRÊA, 2015), equipes multiprofissionais (incluindo enfermeiros, médicos, psicólogos e agentes comunitários de saúde) (FAVORETO; CABRAL, 2009; PEREIRA ET AL., 2007) e profissionais de saúde mental (JORGE ET AL., 2012), com ou sem a presença de profissional observador.

Para a concretização dos objetivos dos grupos, os profissionais da saúde e os participantes discutiram temas específicos, sendo que em cinco estudos esses foram definidos no decorrer dos encontros, de acordo com as necessidades dos participantes (DUTRA; CORRÊA, 2015; FAVORETO; CABRAL, 2009; FIGUEROA; GUERRA; GALlEGOS, 2010; JARDIM ET AL., 2007; PEREIRA ET AL., 2007); um deles, com o uso de tema gerador. Em três estudos, a definição dos temas foi anterior ao início do grupo, com o acordo entre todos (ALMEIDA; SOARES, 2010; ROTOLI ET AL., 2012; SOARES; FERRAZ, 2007). E em um, os temas foram recomendados pelo programa ao qual o grupo pertencia, Programa Nacional de
Controle do Tabagismo (LUCCHESE et AL., 2013). Destaca-se que, em todos os estudos de promoção da saúde, os temas foram construídos no decorrer da intervenção.

Um tema que se sobressaiu nos estudos foi a 'alimentação'. No estudo de Figueroa, Guerra e Gallegos (2010), ela era o centro da discussão, dado que a tarefa do grupo era trabalhar o consumo da alfafa, mas, devido à complexidade do tema, outras discussões foram incorporadas. Já nos estudos de Almeida e Soares (2010), Favoretto e Cabral (2009) e Lucchese et al., (2013), que tinham outros temas centrais, a 'alimentação' emergiu a partir dos participantes, sobretudo a dificuldade da mudança de hábito alimentar.

Para a discussão dos temas, cinco estudos mencionaram o emprego de estratégias educativas (ALMEIDA; SOARES, 2010; JARDIM ET AL., 2007; PEREIRA ET AL., 2007; ROTOLI ET AL., 2012; SOARES; FERRAZ, 2007), como círculo, dinâmica, festa comemorativa, oficina, painel, folder, cartilha, atividade de alongamento, jogo e vídeo. Aponta-se que poucos estudos as descreveram, sendo que dos cinco estudos, quatro eram do tipo estudo de caso/relato de experiência, porém essa descrição foi feita de forma generalizada. Uma exceção a isso foi o estudo de Rotoli et al. (2012), que apresentou as particularidades dos encontros, os temas e as estratégias educativas detalhadamente.

Em relação à avaliação dos grupos, de acordo com a intencionalidade, a observação do profissional da saúde foi o método mais aplicado. A observação do processo grupal foi realizada em oito estudos (ALMEIDA; SOARES, 2010; CASSOL ET AL., 2012; DUTRA; CORRÊA, 2015; FIGUEROA; GUERRA; GALLEGOS, 2010; LUCCHESE ET AL., 2013; PEREIRA ET AL., 2007; ROTOLI ET AL., 2012; SOARES; FERRAZ, 2007) e a observação individual (mãe-filha) em um (JARDIM ET AL., 2007). Entrevista com os participantes pós-grupo foi realizada em três estudos (ALMEIDA; SOARES, 2010; CASSOL ET AL., 2012; FAVORETO; CABRAL, 2009); aplicação de questionário pré e pós-grupo, em dois (COLOSIO ET AL., 2007; LUCCHESE ET AL., 2013); e grupo focal, em um (JORGE ET AL., 2012). Salienta-se que os estudos de 
promoção da saúde empregaram a observação; e os de prevenção e de tratamento, os outros métodos ou a associação entre eles. Para apoiar a avaliação do grupo, no sentido de registrar o acontecimento e analisar a intervenção, observou-se que três estudos utilizaram a elaboração de narrativa, seja individual ou grupal. Quando individual, o ponto de partida foi a entrevista (FAVORETO; CABRAL, 2009); e quando grupal, foi a observação (LUCCHESE ET AL., 2013) ou o grupo focal (JORGE ET AL., 2012).

Apesar de pouco presentes, averiguaram-se indicadores quantitativos e qualitativos de avaliação. O primeiro, por meio da avaliação de grau de dependência, de atitude e de conhecimento, provida pela aplicação de questionário pré e pós-grupo (COLOSIO ET AL., 2007; LUCCHESE ET AL., 2013). E o segundo, por meio de vetores de avaliação do GO aplicados a partir da observação do processo grupal (LUCCHESE ET AL., 2013; SOARES; FERRAZ, 2007). Foi possível perceber que, quando o foco é o indivíduo, a avaliação tende a ser realizada por abordagens quantitativas, e quando o foco é o processo grupal, o uso de abordagens qualitativas é necessário.

\section{Discussão}

A teoria do GO, quando introduzida no Brasil, na década de 1970, foi majoritariamente instituída em três estados: Bahia, São Paulo e Rio Grande do Sul. O destaque do último estado, no presente estudo, pode ser consequência da existência de institutos de pesquisas sobre o autor Enrique PichonRivière, até os dias atuais, diferentemente do que ocorre com os dois primeiros estados.

Verificou-se a relevância dos estudos do tipo estudo de caso/relato de experiência, que consistem em pesquisas profundas e exaustivas de um ou poucos objetos, de maneira que permitam seu amplo e detalhado conhecimento (GIL, 2007). Pode-se dizer que, com esses estudos, mesmo que descritos de forma generalizada, pesquisadores e/ou profissionais da saúde adquirem a possibilidade de reproduzir a intervenção ou de respaldar seus grupos, diferentemente do que acontece com os outros estudos aqui analisados.

A proeminência do cuidado de usuários portadores de doenças mentais, particularmente de usuários de álcool e outras drogas, dentro de serviços especializados é decorrência de a teoria ter sido elaborada por um psiquiatra e psicanalista, que a aplicou em grupos de pacientes de hospitais psiquiátricos. Por outro lado, como o GO é flexível para grupos que possuem vínculo e objetivo comum (PICHON-RIVIĖRE, 2009), percebe-se a ampliação de seu uso em relação à população e ao contexto, sendo que atualmente há um incentivo a este uso dentro dos serviços de saúde de atenção básica (BRASLL, 2014A, 2014B), o que tem contribuído para a ampliação aqui constatada.

O panorama do planejamento de GO evidencia que o autor criador da teoria é usado como referencial teórico, mas também mostra a presença de outros autores mais contemporâneos, talvez pelo fato de estes proverem uma leitura mais criativa e apresentarem a teoria direcionada para públicos específicos, como se vê no caso dos portadores de doenças crônicas. A configuração de grupos homogêneos é defendida, por exemplo, pelos autores Mello Filho (2007) e Osório (1986), que referem que a condição comum entre os participantes provoca identificação, união, suporte social e afirmação da individualidade, o que resulta em melhorias na saúde. Pichon-Rivière (2009) também argumenta esta vantagem em grupos homogêneos, mas, age da mesma forma em relação a grupos heterogêneos, aludindo que a diferença é enriquecedora para os participantes, pois eles podem ser com os outros sem terem que ser como os outros para serem aceitos.

Chamam a atenção os grupos denominados 'grupo operativo de ensino-aprendizagem' e 'grupo operativo de reflexão'. Zimerman (2000) justifica tal classificação pela importância da presença de reflexão dentro dos grupos, que possuem o propósito da informação e da formação. Por outro lado, percebe-se que a reflexão e 
a aprendizagem são intrínsecas ao GO (PICHONRIVIÈRE, 2009), não necessitando de ênfase. Observou-se que os estudos que referenciaram Gayotto (2003) foram os únicos que utilizaram os vetores de avaliação do GO, talvez pelo fato de a autora apresentar a teoria de maneira ampliada para contexto e população, diferentemente dos outros três autores, que enfatizaram o aspecto terapêutico da intervenção.

Sugere-se que o uso dos novos referenciais teóricos pode gerar mudanças positivas ou negativas na intervenção, a depender do objetivo do grupo. Porém, constatou-se que a teoria de Pichon-Rivière (2009) é favorável quando o grupo tem por intenção a transformação de conhecimentos, relações e identidades, tornando o autor um referencial teórico primordial no planejamento de grupos que buscam o cuidado integral, principalmente a promoção da saúde dos participantes.

Constatou-se, nos estudos analisados, que o GO pode ser utilizado na prevenção de riscos e doenças e no tratamento de cuidados específicos, em particular, nas doenças crônicas, que requerem mudanças de hábitos de vida e de cuidados contínuos. Logo, o GO é uma teoria que instiga mudanças, autocuidado e melhor convivência com a doença (ALMEIDA; SOARES, 2010; FAVORETO; CABRAL, 2009). Entretanto, destacou-se, nos estudos, o uso do GO na promoção da saúde associada à educação em saúde e à educação alimentar e nutricional, visto que, na área da saúde, essa associação é atual, apesar de o GO ter sido elaborado na década de 1940. Recentemente, Oliveira et al. (2016), em um grupo de promoção da saúde de cuidadores de crianças deficientes, em uma UBS, confirmaram que a teoria é favorável para a educação em saúde, em virtude de estimular o processo de aprendizagem.

A promoção da saúde tem, entre seus objetivos, o estímulo da autonomia em saúde, transversalizada nas experiências cotidianas, singulares e subjetivas, que são potencializadas no conviver e no aprender com o outro. Assim, o GO é declarado como uma teoria promotora da saúde, mesmo quando se tem a intenção de tratar doenças (FAVORETO; CABRAL, 2009; JORGE ET AL., 2012), como as doenças crônicas ou a dependência de álcool e outras drogas, e de prevenir riscos, como o tabagismo.

Vale ressaltar, ainda, que a Educação Alimentar e Nutricional (EAN), na tentativa de se prevenir e controlar os problemas alimentares e nutricionais contemporâneos, tem sido renomada como estratégia fundamental para a promoção da alimentação adequada e saudável e, portanto, da saúde (BRASIL, 2012). Ademais, a EAN vem se destacando nas políticas públicas de saúde (BRASIL, 2012, 2014A, 2014B) e nas pesquisas que visam à promoção da saúde (BOTELHO ET AL., 2016; ESTEVES; BeNTO, 2015). Como exemplo disto, cita-se o estudo de Esteves e Bento (2015), que usou o GO para promover a alimentação materno infantil de um grupo de gestantes, em uma UBS, e notou aumento da segurança das participantes em suas escolhas alimentares, demostrando o êxito do GO nas questões que envolvem a EAN. O estudo citado não foi identificado na base de dados aqui utilizada, mas reforça os dados encontrados.

Em relação às regras de organização dos grupos, foi observada a existência de distintas possiblidades, sendo que essas dependerão do objetivo do grupo. No entanto, compreende-se que grupos pequenos, de 5 a 12 participantes, são os privilegiados com o uso da teoria, pois favorecem a reflexão e a criatividade (AFONSO, 2010). Realça-se a presença dos enfermeiros como coordenadores da ação, o que pode ser resultado da vivência deles como participantes de GO na formação acadêmica, conforme verificado por Correa, Melo e Souza e Saeki (2005), Moura e Caliri (2013) e Oliveira e Ciampone (2008), sendo a vivência um disparador de interesse em reproduzi-la nos serviços de saúde. Esta vivência pode ser inspiradora para a formação de outros profissionais, visto que a coordenação de GO também é atribuída a equipes multiprofissionais. Outra possibilidade é que a divulgação do conhecimento no âmbito da análise das práticas no campo da saúde mental ainda ocorra, preferencialmente, por meio de livros e de contatos pessoais, como 
grupos de estudos e cursos de difusão, ou seja, não aconteça na base de dados adotada ou por meio de ensaios teóricos, excluídos neste estudo.

Na definição dos temas a serem discutidos nos grupos, verificam-se três possibilidades: definição a partir das necessidades, incluindo o uso de tema gerador; cronograma definido anteriormente, em relação ao início do grupo; e definição externa. Todavia, independentemente do caminho tomado, considera-se a inclusão das necessidades dos participantes um fator primordial no GO. Desta forma, mesmo na existência de um cronograma definido externamente ao serviço de saúde, é o profissional que levará tal inclusão no momento da ação, uma vez que a demanda das necessidades de saúde, dependente da percepção e da interpretação de todos os envolvidos, não segue uma lógica linear (AFONSO, 2010). Distingue-se o uso de tema gerador, conceito operacional da educação dialógica elaborada por Paulo Freire, que é um tema escolhido democraticamente entre todos e é condutor da reflexão (DUTRA; CORRÊA, 2015). Indica-se uma fusão positiva entre o GO e a educação dialógica, posto que haja entre eles uma reciprocidade e uma complementaridade do aprender e do ensinar (AFONSO; VIEIRA-SILVA; ABADE, 2009).

A 'alimentação' foi tema recorrente nos estudos, porém apareceu como um obstáculo para as mudanças de hábitos. Dado que trabalhar a alimentação envolve dimensões no campo da cognição, da percepção, do afeto, das relações e das habilidades pessoais (BOOG, 2013), relacionar a EAN e o GO dentro de um grupo específico de alimentação e nutrição pode ser propício para superar tal obstáculo.

Os temas, em alguns dos estudos, foram tratados com o emprego de estratégias educativas, as quais são técnicas que favorecem a sensibilização, a expressão e a comunicação, consideradas táticas eficientes de aprendizagem (AFONSO, 2010). Contudo, poucos estudos as descrevem, ou por elas não serem necessárias ou por não serem valorizadas no desenvolvimento de GO, ao contrário do que acontece nos grupos educativos encontrados nos serviços de saúde, nos quais profissionais preocupam-se mais com as estratégias educativas do que com a teoria grupal, perfazendo, muitas vezes, uma participação limitada dos usuários (BOTELHO ET AL., 2016)

$\mathrm{Na}$ avaliação dos grupos, a observação dos profissionais como método de avaliação da intervenção foi o mais empregado. A observação é uma análise do coordenador ou do observador, a partir das ações dos participantes, fundamentada nos referenciais teóricos do avaliador. Já a entrevista ou a aplicação de questionário ou o grupo focal são métodos de análise dos participantes sobre a vivência no grupo. Todos são propícios para a avaliação de um GO, mas adquirem maior magnitude quando realizados em conjunto, pois expressam o olhar do profissional e a vivência do participante. Além disto, a relação entre, por exemplo, observação e entrevista permite analisar não somente o processo grupal, mas também o acompanhamento individual, uma vez que, segundo Pichon-Rivière (2009), dentro de um grupo, os participantes possuem um duplo investimento: buscam o reconhecimento pela igualdade grupal e o prestígio como indivíduo.

Notou-se uma lógica entre os métodos e indicadores utilizados e os objetivos dos grupos: os que tinham a intencionalidade de prevenir riscos e doenças foram avaliados por indicadores individuais de atitudes e de conhecimentos, enquanto os de tratamento utilizaram narrativas - individuais ou grupais - para avaliar a ressignificação do cuidado. Entretanto, o fato de poucos estudos utilizarem indicadores de avaliação permite afirmar que há uma fragilidade na avaliação dos GO dentro dos serviços de saúde. Isto é consequência, possivelmente, da incorporação de indicadores quantitativos na prática dos profissionais da saúde, os quais são insuficientes para delinear a avaliação do processo de aprendizagem dos GO. Em alternativa, essa avaliação tem 
sido retratada por pesquisas de abordagem qualitativa, nas quais profissionais e, principalmente, usuários expressam seus sentimentos, pensamentos e opiniões, que podem fomentar o planejamento dos GO, substancializando os indicadores qualitativos. Assinala-se que esses indicadores, no que se refere aos indivíduos, podem ser representados pelos níveis de empoderamento e de autonomia em saúde, que envolvem, de acordo com Bravo et al. (2016), conhecimento, atitude, habilidade, autopercepção, autocuidado, decisão compartilhada, qualidade de vida, bem-estar/satisfação com a vida etc.

\section{Conclusões}

A revisão possibilitou a construção de um panorama de planejamento de GO, que poderá auxiliar os profissionais da saúde na tomada de decisões quanto ao planejamento de seus grupos. A escolha do uso da teoria pelo profissional ou pelo serviço requer a adoção de referenciais teóricos. Nesse sentido, apurou-se o uso de referenciais mais contemporâneos do que os de Pichon-Rivière, o que poderá viabilizar uma flexibilização do uso do GO. Em contrapartida, o uso desses referenciais também pode distanciar a intervenção da essência da teoria, dado que apenas dois estudos utilizaram seus vetores de avaliação. A transformação do conhecimento é frutífera, mas compreende-se que a combinação entre o originário e a variação é essencial para o desenvolvimento de grupos que almejam a construção do vínculo, do protagonismo e da autonomia em saúde dos participantes.

Os elementos do planejamento dos GO foram identificados nos estudos analisados, e foi nos grupos de prevenção e de tratamento que eles apresentaram melhor estrutura, pois se trata de uma atenção consolidada na área da saúde. A promoção da saúde vem ganhando destaque mais recentemente, além de envolver uma multiplicidade de fatores que não se limitam aos serviços e profissionais da saúde, o que dificulta planejar suas ações. Nesta lógica, sugere-se aos profissionais, coordenadores de GO promotores da saúde, que planejem seus grupos com base nas necessidades de saúde, a partir de um diagnóstico de saúde dos participantes ou do território de atuação, reforçado pela discussão com outros profissionais e pelos conhecimentos e experiências prévios, avaliando-os constantemente por meio dos vetores de avaliação do GO e dos níveis de empoderamento e autonomia em saúde.

Por fim, espera-se que este estudo possa ser um estímulo, juntamente com a implementação da Política Nacional da Promoção da Saúde, ao desenvolvimento e à publicação de outros estudos para a consolidação da teoria do GO no âmbito das políticas públicas atuais de saúde, com ênfase na promoção da saúde.

\section{Colaboradores}

VINCHA, K. R. R. contribui na concepção da pesquisa, na análise e interpretação dos dados e na redação do manuscrito. SANTOS, A. de F. contribuiu na coleta de dados, na análise dos dados e na redação do manuscrito. CERVATO-MANCUSO, A. M. contribuiu na concepção da pesquisa, na análise e interpretação dos dados e na aprovação da versão final do manuscrito.

\section{Agradecimentos}

À Coordenação de Aperfeiçoamento de Pessoal de Nível Superior (Capes) pela concessão de bolsa de doutorado à autora Vincha, K. R. R. E ao Programa Unificado de Bolsas de Estudo para Estudantes de Graduação (PUB) da Universidade de São Paulo pela concessão de bolsa à autora Santos, A. F. 


\section{Referências}

AFONSO, M. L. M. Como construir uma proposta de oficina. In: AFONSO, M. L. M. (Org.). Oficinas em dinâmicas de grupo na área da saúde. 2. ed. São Paulo: Casa do Psicólogo, 2010. p. 133-230.

AFONSO, M. L. M.; COUTINHO, A. R. A. Metodologias de trabalho com grupos e sua utilização na área da saúde. In: AFONSO, M. L. M. (Org.). Oficinas em dinâmicas de grupo na área da saúde. 2. ed. São Paulo: Casa do Psicólogo, 2010. p. 59-83.

AFONSO, M. L. M.; VIEIRA-SILVA, M.; ABADE, F. L. O processo grupal e a educação de jovens e adultos. Psicologia em Estudo, Maringá, v. 14, n. 4, p. 707-715, dez. 2009. Disponível em: <http://www.scielo.br/ scielo.php?pid=S1413-73722009000400011\&script=sci abstract\&tlng=pt>. Acesso em: 2 fev. 2017.

ALMEIDA, S. P.; SOARES, S. M. Aprendizagem em grupo operativo de diabetes: uma abordagem etnográfica. Ciência \& Saúde Coletiva, Rio de Janeiro, v. 15, supl. 1, p. 1123-1132, jun. 2010. Disponível em: <http://www.scielo.br/scielo.php?pid=S1413$-81232010000700020 \&$ script $=$ sci_abstract\&tlng $=$ pt $>$. Acesso em: 2 fev. 2017.

BOOG, M. C. F. Educação em Nutrição: integrando experiências. Campinas: Komedi, 2013.

BOTELHO, F. C. et al. Estratégias pedagógicas em grupos com o tema alimentação e nutrição: os bastidores do processo de escolha. Ciência \& Saúde Coletiva, Rio de Janeiro, v. 21, n. 6, p. 1889-1898, jun. 2016. Disponível em: <http://www.scielo.br/scielo.php?pid=S1413$-81232010000700020 \&$ script $=$ sci_abstract $\&$ tlng $=$ pt $>$. Acesso em: 02 fev. 2017.

BRASIL. Ministério da Saúde. Secretaria de Atenção à Saúde. Departamento de Atenção Básica. Estratégias para o cuidado da pessoa com doença crônica. Brasília, DF: Ministério da Saúde, 2014c. Disponível em: <http:// bvsms.saude.gov.br/bvs/publicacoes/estrategias_cuidado_pessoa_doenca_cronica_cab35.pdf $>$. Acesso em: 2 fev. 2017
. Ministério da Saúde. Secretaria de Atenção à Saúde. Departamento de Atenção Básica. Estratégias para o cuidado da pessoa com doença crônica: obesidade. Brasília, DF: Ministério da Saúde, 2014b. Disponível em: <http://bvsms.saude.gov.br/bvs/publicacoes/estrategias_cuidado_doenca_cronica_obesidade_cab38.pdf $>$. Acesso em: 2 fev. 2017.

. Ministério da Saúde. Secretaria de Vigilância em Saúde. Secretaria de Atenção à Saúde. Política Nacional de Promoção da Saúde. Brasília, DF: Ministério da Saúde, 2014a. Disponível em: <http://bvsms.saude.gov. br/bvs/publicacoes/politica_nacional_promocao_saude_pnaps.pdf>. Acesso em: 2 fev. 2017.

Ministério do Desenvolvimento Social e Combate à Fome. Marco de Referência de Educação Alimentar e Nutricional para as Políticas Públicas. Brasília, DF: Ministério da Saúde, 2012. Disponível em: <https://www.ideiasnamesa.unb.br/files/marco_EAN visualizacao.pdf>. Acesso em: 2 fev. 2017.

BRAVO, P. et al. Conceptualising patient empowerment: a mixed methods study. BMC Health Services Research, Londres, v. 15, n. 252, p. 1-14, dez. 2015. Disponível em: <https://www.ncbi.nlm.nih.gov/pmc/ articles/PMC4488113/>. Acesso em: 2 fev. 2017.

BUFREM, L.; PRATES, Y. O saber científico registrado e as práticas de mensuração da informação. Ciência da Informação, Brasília, DF, v. 34, n. 2, p. 9-25, ago. 2005. Disponível em: <http://revista.ibict.br/ciinf/article/ view/1086>. Acesso em: 2 fev. 2017.

CASSOL, P. B. et al. Tratamento em um grupo operativo em saúde: percepção dos usuários de álcool e outras drogas. Revista Gaúcha de Enfermagem, Porto Alegre, v. 33, n. 1, p. 132-138, mar. 2012. Disponível em: < http:// www.scielo.br/scielo.php?script=sci_arttext\&pid =S1983-14472012000100018>. Acesso em: 2 fev. 2017.

CASTANHO, P. Uma introdução aos grupos operativos: teoria e técnica. Vínculo, São Paulo, v. 9, n. 1, p. 47-60, jun. 2012. Disponível em: <http://bases.bireme.br/cgi-bin/wxislind.exe/iah/online/?IsisScript=iah/iah.xis 
$\& \mathrm{src}=$ google $\&$ base $=$ LILACS $\&$ lang $=$ p $\&$ nextAction $=\ln k$ \&exprSearch=693531\&indexSearch=ID $>$. Acesso em: 2 fev. 2017.

CERVATO-MANCUSO, A. M. Elaboração de um programa de educação nutricional. In: DIEZ-GARCIA; CERVATO-MANCUSO (Org.). Mudanças alimentares e educação nutricional. Rio de Janeiro: Guanabara Koogan, 2011. p. 187-197.

COLOSIO, R. et al. Prevenção de infecção pelo HIV por intermédio da utilização do grupo operativo entre homens que fazem sexo com homens, São Paulo, Brasil. Cadernos de Saúde Pública, Rio de Janeiro, v. 23, n. 4, p. 949-959, abr. 2007. Disponível em: <http://www.scielo.br/scielo.php?pid=S0102$-311 X 2007000400022 \&$ script $=$ sci_abstract\&tlng $=p t>$. Acesso em: 2 fev. 2017.

CORREA, A. K.; MELLO E SOUZA, C. B.; SAEKI, T. Transição para o exercício profissional em enfermagem: uma experiência em grupo operativo. Escola Anna Nery, Rio de Janeiro, v. 9, n. 3, p. 421-428, dez. 2005. Disponível em: <http:// www.scielo.br/scielo.php?script=sci_arttext\&pid $=$ S1414-81452005000300010>. Acesso em: 2 fev. 2017.

DUTRA, W. H.; CORREA, R. M. O Grupo Operativo como Instrumento Terapêutico-Pedagógico de Promoção à Saúde Mental no Trabalho. Psicologia: Ciência e Profissão, Brasília, v. 35, n. 2, p. 515-527, jun. 2015. Disponível em: <http://www.scielo.br/scielo. php?pid=S1414-98932015000200515\&script=sci_abstract>. Acesso em: 2 fev. 2017.

ESTEVES, J. M. M.; BENTO, I. C. Promoção da alimentação materno e infantil em um grupo operativo de gestantes. Revista de APS, Juiz de Fora, v. 18, n. 2, p. 213-219, abr./jun. 2015. Disponível em: <https://aps.ufjf. emnuvens.com.br/aps/article/viewFile/2290/880>. Acesso em: 2 fev. 2017.

FAVORETO, C. A. O.; CABRAL, C. C. Narrativas sobre o processo saúde-doença: experiências em grupos operativos de educação em saúde. Interface: Comunicação, Saúde, Educação, Botucatu, v. 13, n. 28, p. 7-18, mar.
2009. Disponível em: <http://www.scielo.br/scielo. php?pid=S1414-32832009000100002\&script=sci abstract\&tlng=pt $>$. Acesso em: 2 fev. 2017.

FERREIRA NETO, J. L.; KIND, L. Promoção da Saúde: Práticas Grupais na Estratégia Saúde da Família. Belo Horizonte: Fapeming, 2011.

FIGUEROA, I. A. V.; GUERRA, J. G.; GALLEGOS, N. G. Proceso de grupo durante la realización de un proyecto educativo nutricional comunitario. Revista Cubana de Salud Pública, Ciudad de La Habana, v. 36, n. 2, p. 148-155, maio/jun. 2010. Disponível em: <http://scielo.sld.cu/scielo.php?script=sci_arttext\&pid =S0864-34662010000200008>. Acesso em: 2 fev. 2017.

GAYOTTO, M. L. C. Avaliando o desempenho grupal. In: GAYOTTO, M. L. C. et al. (Org.). Liderança II: aprenda a coordenar grupos. Petrópolis: Vozes, 2003. p. 185-219.

GIL, A. C. Como elaborar projetos de pesquisa. 4. ed. São Paulo: Atlas, 2007.

JARDIM, F. C. et al. Intervenção psicoterápica de grupo com pais e bebês: relato de um caso. Revista de Psiquiatria do Rio Grande do Sul, Porto Alegre, v. 29, n. 3, p. 321-323, set./dez. 2007. Disponível em: <http:// www.scielo.br/scielo.php?script=sci_arttext\&pid =S0101-81082007000300012>. Acesso em: 2 fev. 2017.

JORGE, M. S. B. et al. Experiências com a gestão autônoma da medicação: narrativa de usuários de saúde mental no encontro dos grupos focais em centros de atenção psicossocial. Physis: Revista de Saúde Coletiva, Rio de Janeiro, v. 22, n. 4, p. 1543-1561, jan. 2012. Disponível em: <http://www.scielo.br/scielo. php?pid=S0103-73312012000400015\&script=sci abstract\&tlng=pt $>$. Acesso em: 2 fev. 2017.

LUCCHESE, R. et al. A tecnologia de grupo operativo aplicada num programa de controle do tabagismo. Texto \&t Contexto Enfermagem, Florianópolis, v. 22, n. 4, p. 918-926, dez. 2013. Disponível em: <http://www.scielo.br/pdf/tce/v22n4/07.pdf>. Acesso em: 2 fev. 2017. 
MELLO FILHO, J. Grupo e corpo: psicoterapia de grupo com pacientes somáticos. São Paulo: Casa do Psicólogo, 2011.

MELO, L. P. Análise biopolítica do discurso oficial sobre educação em saúde para pacientes diabéticos no Brasil. Revista Saúde e Sociedade, São Paulo, v. 22, n. 4, p. 1216-1225, dez. 2013. Disponível em: <https://www. revistas.usp.br/sausoc/article/viewFile/76514/80261>. Acesso em: 2 fev. 2017

MENEZES, K. K. P.; AVELINO, P. R. Grupos operativos na Atenção Primária à Saúde como prática de discussão e educação: uma revisão. Cadernos de Saúde Pública, Rio de Janeiro, v. 24, n. 1, p. 124-130, mar. 2016. Disponível em: <http://www.scielo.br/pdf/cadsc/ v24nl/1414-462X-cadsc-24-1-124.pdf>. Acesso em: 2 fev. 2017.

MOURA, E. C. C.; CALIRI, M. H. L. Simulação para desenvolvimento da competência clínica de avaliação de risco para úlcera por pressão. Acta Paulista de Enfermagem, São Paulo, v. 26 n. 4, p. 369-375, 2013. Disponível em: <http:// www.scielo.br/scielo.php?script=sci_arttext\&pid =S0103-21002013000400011>. Acesso em: 2 fev. 2017

OLIVEIRA, D. M. et al. O grupo operativo como instrumentos de aprendizagem do cuidado por mães de filhos com deficiência. Escola Anna Nery, Rio de Janeiro, v. 20, n. 3, p. 1-7, jun. 2016. Disponível em: <http://www.scielo.br/scielo.php?pid=S1414$-81452016000300221 \&$ script $=$ sci_abstract\&tlng $=$ pt $>$.

Acesso em: 2 fev. 2017.

\section{OLIVEIRA, R. A.; CIAMPONE, M. H. T.}

Qualidade de vida de estudantes de enfermagem: a construção de um processo e intervenções. Revista da Escola de Enfermagem USP, São Paulo, v. 42, n. 1, p. 57-65, mar. 2008. Disponível em: <http://webcache.googleusercontent.com/ search?q=cache:8601QVylvz8J:www.revistas.usp.br/ reeusp/article/download/41706/45319+\&cd=1\&hl=pt-
-BR\&ct=clnk\&gl=br>. Acesso em: 2 fev. 2017.

OSÓRIO, L. C. Grupoterapia hoje. Porto Alegre: Artes Médias, 1986

PEREIRA, Q. L. C. et al. Processo de (re)construção de um grupo de planejamento familiar: uma proposta de educação popular em saúde. Texto \& Contexto Enfermagem, Florianópolis, v. 16, n. 2, p. 320-325, jun. 2007. Disponível em: <http://www.scielo.br/pdf/tce/ v16n2/a16v16n2>. Acesso em: 2 fev. 2017.

PICHON-RIVIÈRE, E. O processo grupal. 8. ed. São Paulo: Martins Fontes, 2009.

ROTOLI, A. et al. Grupos operativos: Estrategia para el cuidado de enfermería en un centro de atención psicosocial. Index de Enfermería, Granada, v. 21, n. 4, p. 229-233, out./dez. 2012. Disponível em: <http:// scielo.isciii.es/scielo.php?script=sci_arttext\&pid =S1132-12962012000300011 >. Acesso em: 2 fev. 2017.

SOARES, S. M.; FERRAZ, A. F. Grupos operativos de aprendizagem nos serviços de saúde: sistematização de fundamentos e metodologias. Escola Anna Nery, Rio de Janeiro, v. 11, n. 1, p. 52-57, mar. 2007. Disponível em: $<$ http://www.scielo.br/pdf/ean/vllnl/vllnla07.pdf>. Acesso em: 2 fev. 2017.

VINCHA, K. R. R. et al. Grupos de educação nutricional em dois contextos da América Latina: São Paulo e Bogotá. Interface: Comunicação, Saúde, Educação, Botucatu, v. 18, n. 50, p. 507-520, set. 2014. Disponível em: <http://www.scielo.br/scielo.php?pid=S1414$-32832014000300507 \&$ script $=$ sci_abstract\&tlng=pt $>$. Acesso em: 2 fev. 2017.

ZIMERMAN, D. E. Fundamentos básicos das grupoterapia. 2. ed. Porto Alegre: Artmed, 2000.

\footnotetext{
Recebido para publicação em março de 2017 Versão final em julho de 2017

Conflito de interesses: inexistente

Suporte financeiro: não houve
} 